\title{
A multi-layer perceptron for scheduling cellular manufacturing systems in the presence of unreliable machines and uncertain cost
}

\begin{abstract}
In this paper, a new method is proposed for short-term period scheduling of dynamic cellular manufacturing systems in the presence of bottleneck and parallel machines. The aim of this method is to find best production strategy of in-house manufacturing and outsourcing in small and medium scale cellular manufacturing companies. For this purpose, a multi-period scheduling model has been proposed which is flexible enough to be used in real industries. To solve the proposed problem, a number of metaheuristics are developed including Branch and Bound; Simulated Annealing algorithms; Fuzzy Art Control; Ant Colony Optimization and a hybrid Multi-layer Perceptron and Simulated Annealing algorithms. Our findings indicate that the uncertain condition of system costs affects the routing of product parts and may induce machine-load variations that yield to cell-load diversity. The results showed that the proposed method can significantly reduce cell load variation while finding the best trading off values between in-house manufacturing and outsourcing.
\end{abstract}

Keyword: Design of manufacturing; Production system optimization; Modeling and simulation 